\title{
Feasibility of innovative dietary assessment in epidemiological studies using the approach of combining different assessment instruments
}

\author{
Anne-Kathrin Illner ${ }^{1, *}+$, Ulrich Harttig ${ }^{1}$, Gianluca Tognon ${ }^{2,3}$, Domenico Palli ${ }^{2}$, \\ Simonetta Salvini ${ }^{2}$, Eugenia Bower ${ }^{4}$, Pilar Amiano ${ }^{4}$, Taie Kassik ${ }^{5}$, Andres Metspalu ${ }^{5}$, \\ Dagrun Engeset ${ }^{6},{ }^{2}$ Eiliv Lund ${ }^{6}$, Heather Ward $^{7}$, Nadia Slimani $^{8}$, Manuela Bergmann $^{1}$, \\ Karen Wagner ${ }^{1}$ and Heiner Boeing ${ }^{1}$ \\ 'Department of Epidemiology, German Institute of Human Nutrition Potsdam-Rehbruecke, Nuthetal, Germany: \\ ${ }^{2}$ Molecular and Nutritional Epidemiology Unit, Cancer Prevention and Research Institute (ISPO), Florence, \\ Italy: ${ }^{3}$ Public Health Epidemiology Unit, Department of Public Health and Community Medicine, University of \\ Gothenburg, Gothenburg, Sweden: ${ }^{4}$ Public Health Division of Gipuzkoa, San Sebastian, Basque Government, \\ CIBER Epidemiology and Public Health, CIBERESP, Spain: ${ }^{5}$ University of Tartu and Estonian Biocentre, Tartu, \\ Estonia: ${ }^{6}$ Department of Community Medicine, University of Tromsø, Tromsø, Norway: ${ }^{7}$ Department of Public \\ Health and Primary Care, MRC Centre for Nutritional Epidemiology in Cancer Prevention and Survival, \\ University of Cambridge, Cambridge, UK: ${ }^{8}$ Dietary Exposure Assessment Group, International Agency for \\ Research on Cancer, WHO, Lyon, France
}

Submitted 7 April 2010: Accepted 23 November 2010: First published online 9 March 2011

\begin{abstract}
Objective: To assess the feasibility of combining short-term and long-term dietary assessment instruments as new concept for improving usual dietary intake assessment on the individual level.

Design: Feasibility study of completing three $24 \mathrm{~h}$ dietary recalls (24-HDR) and a self-administered food propensity questionnaire (FPQ). The 24-HDR was conducted by monthly telephone interviews, using EPIC-SOFT software. The FPQ was completely standardized across cohorts and offered either as a web-based tool or in paper format.

Setting: Random sample derived from five ongoing European cohort studies (EPIC-San Sebastian, EPIC-Florence, EPIC-Potsdam, Estonia Genome Center (EGC) and Norwegian Women and Cancer study (NOWAC)).

Subjects: A total of 400 participants.

Results: Overall, the total participation rate for the present study was $65.3 \%$ ( $n$ 261). On average, completion of the 24-HDR was highest for the first 24-HDR $(63.0 \%)$ and decreased slightly for the second $(60.3 \%)$ and third 24-HDR $(56 \cdot 3 \%)$. The proportions of selecting the web-based FPQ varied among the study centres, with the highest in EGC (92.9\%) and NOWAC (70.0\%) and the lowest in EPIC-San Sebastian (25.5\%) and EPIC-Potsdam (33.9\%). Web users rarely requested support and were younger and more highly educated than those who completed the paper format.

Conclusions: The present study supports the feasibility of a combined application of three 24-HDR and an FPQ in culturally different populations. The varying acceptance of the web-based instrument across populations requires a flexible application of assessment instruments.
\end{abstract}

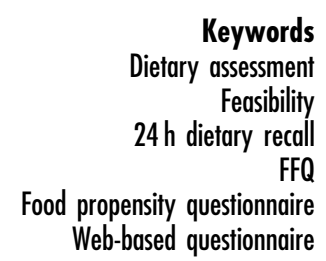

Keywords

Feasibility

FFQ

Web-based questionnaire
Findings of validation and comparative risk studies indicate that the standard dietary assessment instrument in large-scale prospective studies since the 1980s, the FFQ,

$\uparrow$ Present affiliation and correspondence address: Dietary Exposure Assessment Group, International Agency for Research on Cancer, WHO, 150 Cours Albert Thomas, Lyon 69372, France. might not provide the dietary data needed to investigate diet-disease relationships properly ${ }^{(1,2)}$. Therefore, researchers argue about whether to replace or supplement the errorprone FFQ by short-term dietary assessment instruments, such as food records or repeated $24 \mathrm{~h}$ dietary recalls $(24-\mathrm{HDR})^{(3)}$. Compared with an FFQ that enquires about diet over a relatively long period of time, short-term instruments 
may have a potential cognitive advantage and provide quantitative and detailed dietary data ${ }^{(4)}$. Furthermore, especially the 24-HDR has the ability to obtain standardized dietary information in culturally diverse groups or multi-centre studies ${ }^{(5)}$. However, the variance of dietary estimates based on repeated 24-HDR is inflated by intraindividual variance ${ }^{(6,7)}$. In addition, the estimation of dietary intakes of irregularly consumed foods, if not supplemented, may be biased, since the 24-HDR usually includes a substantial proportion of participants reporting zero intakes during the recorded days. Long-term instruments such as FFQ or food propensity questionnaires (FPQ - an FFQ that considers only the frequency of consumption) may have the potential to identify habitual users of such foods ${ }^{(8)}$. Recent statistical advances in estimating usual dietary intakes therefore combine the advantages of both quantitative and rich 24-HDR data and non-quantitative FFQ/FPQ information about habitual consumers while minimizing their limitations (e.g. adjusting for intra-individual variation in the 24-HDR data) ${ }^{(9)}$. Promising results have already been obtained from methodological and simulation studies showing an increased precision when including the reported frequency of consumption on an FFQ or FPQ as a covariate in the model (J Haubrock, U Nöthlings, J-L Volatier et al., unpublished results) ${ }^{(10,11)}$. Nevertheless, future challenges of these statistical combination methods include exploring empirically the optimal number of repeated short-term measurements, and also testing the feasibility of data collection, including the mode of enquiry to collect multiple dietary information, especially in culturally diverse populations. Particularly, FFQ or FPQ are suited to be adapted to Internet technologies and to being applied as web-based applications ${ }^{(12,13)}$. However, knowledge about their practical feasibility and acceptance is still limited.

In the present paper, we report about the feasibility of applying three monthly telephone-administered 24-HDR in conjunction with a standardized FPQ in a multi-centre pilot study involving five ongoing European cohort populations. The FPQ (hereafter named European Food Propensity Questionnaire (EFPQ)) was created to be selfadministered and available as both web- and paper-based versions. To evaluate the feasibility of the approach, we present response and participation rates, reasons for non-participation, percentages of completion of the instruments, number of contact attempts and data automatically recorded by web analysis.

\section{Subjects, study design and methods}

\section{Subjects and study design}

Between January and April 2009, a random sample of 400 active study participants was generated from five ongoing cohorts, according to age ranges and sex distributions in the vital source cohorts. An upper age limit of 75 years was applied. Thus, eighty participants were recruited from the European Prospective Investigation into Cancer and Nutrition (EPIC)-San Sebastian, Spain (vital source population: $n 7973$; 3833 men and 4140 women), EPICFlorence, Italy ( $n$ 13597; 3514 men and 10083 women), EPIC-Potsdam, Germany ( $n$ 25784; 10335 men and 15449 women) and the Norwegian Women and Cancer study, Norway (NOWAC; 165772 women). In the cohort of the Estonian Genome Center (EGC), University of Tartu, Estonia, eligible subjects were invited during the ongoing baseline recruitment of participants into the cohort. In November 2010, EGC included almost 50000 men and women. All cohorts were based on the general adult populations residing in a given geographical area.

Subjects were invited to complete the single, self-administered EFPQ, either in web-based (web-EFPQ) or paper format (paper-EFPQ), and to answer to the three nonconsecutive, unannounced 24-HDR telephone interviews. Subjects were contacted by regular mail. The mail included the study invitation letter, information material and a response form on which reasons for non-participation, preferences for the EFPQ-administration modes and for the timing of the 24-HDR could be indicated (except in NOWAC, in which questions about non-participation were not permitted by the local privacy law). Furthermore, userspecific instructions with individual username and password for the web-EFPQ were provided to each participant to guarantee data protection. Consent of participation was obtained when subjects accepted the invitation to participate in the pilot study by either sending back the response sheet or directly using the web-EFPQ. Responders with preferences for the paper-EFPQ received a printed personal questionnaire by mail. Participants were offered support on request for both administration modes. To measure the acceptance of the web-EFPQ in each study centre, users were encouraged to complete a web-based brief evaluation questionnaire that enquired their opinion on the clarity of questions and explanations, visual elements, user friendliness, time needed to complete the questionnaire and difficulties experienced in estimating their food intake. A six-item Likert scale from $1=$ brilliant to $6=$ abysmal was offered to rate each question and a free text field was given to add comments and suggestions. There was no evaluation questionnaire administered for the paper-EFPQ. After having completed the EFPQ, a pilot study-specific booklet was mailed in order to help subjects during the three 24-HDR in quantifying their food consumption. An attempt to contact those subjects who answered the web-EFPQ for the first 24-HDR was made no earlier than $5 \mathrm{~d}$ after mailing of the picture booklet, whereas those who preferred the paper-EFPQ were immediately contacted after the return of the completed questionnaire. The 24-HDR, randomly assigned by day of the week, was collected approximately 1 month apart from February to September 2009. In most centres, food intake 
on Saturday was assessed on Monday. For each subject's 24-HDR, contact attempts were documented.

To address potential sources of bias, an explicit study protocol for quality control was designed ensuring a standardized data collection and study management across the centres. Necessary deviations from the common study protocol regarded a different sampling procedure in EGC. Because of the ongoing recruitment, eligible Estonian subjects were asked whether they were interested to participate in the pilot study during the recruitment examinations. After a short oral explanation, subjects received a closed envelope containing the study material and signed the consent of agreement or refused to participate. The study was approved by the responsible ethics committee of the participating centres.

\section{Dietary assessment instruments}

The EPIC-SOFT program (Dietary Exposure Assessment Group, International Agency for Research on Cancer/ WHO, Lyon, France) was used for the administration of the 24-HDR. In Europe, the multi-language EPIC-SOFT is the primary software aimed at assessing detailed and quantified dietary data of a single day in a standardized manner ${ }^{(5,14)}$. Country-specific versions were available in all centres conducting the pilot study, except in EGC, where the English version was used. In the 24-HDR interviews, trained personnel asked subjects to report all food and beverages consumed during the day before the interview. The pilot study-specific picture booklet contained a selection of photographs of portion sizes and household measures (e.g. mugs, glasses and bowls) from the original EPIC-SOFT picture booklet.

The multilingual EFPQ was based on an existing validated FFQ covering 102 typical German food items that was applied in the EPIC-Potsdam cohort ${ }^{(15)}$. The EFPQ was developed using a web-based portal that allowed nutritionists of the pilot study centres to review and modify an English translated version of the German FFQ regarding its adequacy in reflecting the dietary habits of the selected European countries. The experts being familiar with the usual dietary habits in the local population under study could decide online with predefined choices whether or not each item should be kept, modified to a country-specific food or deleted. Additional comments could be entered into free text areas at the bottom of each web page. The common English version was then translated into the local languages: Italian, Norwegian, Basque, Spanish, Estonian and German. The final EFPQ enquired about the frequency of consumption of 116 foods and beverages during the previous 12 months. Portion sizes for most food items were graphically displayed with pictograms. Additional questions were asked about food preparation practices, specifications of several food items (e.g. preferred fat content, additions to hot beverages, such as dairy creamer) and the use of nutritional supplements and medications. Furthermore, summary questions about general consumption patterns and questions about body weight and body height were included. The web-based EFPQ is publicly available at https://nugo.dife.de/efbo/portal/en.

\section{Statistical analysis}

All analyses, unless otherwise specified, were performed after stratification by pilot study centre and ordered according to a geographical south-north gradient.

To investigate the feasibility and acceptance of the approach of combining instruments, overall and centrespecific numbers and percentages of responders, participants and non-participants were computed (response rate and participation rate). Participants were those who completed the EFPQ in order to take into account the initial adherence of subjects to the complete study protocol. Demographic and other lifestyle characteristics of participants and non-participants in each pilot study centre were displayed as arithmetic means and SD (for continuous variables) and frequencies (for categorical variables). Age at enrolment in the pilot study, sex and educational attainment were derived from the baseline assessment of each cohort and collected through standardized questionnaires ${ }^{(16-18)}$. Pilot study enrolment was defined as the date of completion of the EFPQ for participants and date of invitation for non-participants. BMI (calculated as weight in kilograms divided by the square of height in metres $\left(\mathrm{kg} / \mathrm{m}^{2}\right)$, obtained from self-reported or measured data (according to study centre) and smoking status were obtained from the most recent data assessment of each cohort. The time interval varied between lifestyle data collection and pilot study by centre from $1 \mathrm{~d}$ (only for EGC, where recruitment was ongoing) to 6 years. Reasons for non-participation, percentage of completion of the 24-HDR and the administration modes of the EFPQ and the mean number of contact attempts for each 24-HDR were analysed. To specifically assess the practical feasibility of the web-EFPQ data from the evaluation questionnaire, further background data that were automatically recorded by the software when the website was used were also evaluated. Demographic and lifestyle characteristics of subjects with preferences for the web- or paper-EFPQ were displayed. Differences were tested for statistical significance with the unpaired differences $t$ test (for continuous variables) and $\chi^{2}$ test (for categorical variables). $P$ values were two-sided and a significance level of $P<0.05$ was applied. All analyses have been performed using the SAS statistical software package version 9.13 (SAS Institute, Cary, NC, USA).

\section{Results}

Of the 400 eligible participants, 350 responded (overall response rate: $87.5 \%$ ). The centre-specific response rate was $75.8 \%$ in NOWAC ( $n$ 63), $83.8 \%$ in EPIC-Florence ( $n 67), 87.5 \%$ in EPIC-San Sebastian ( $n 70$ ), $92.5 \%$ in EPICPotsdam ( $n 74)$ and $95.0 \%$ in EGC $(n 76)$. Where reported 
on the response form, reasons for non-participation included no interest ( $n$ 16), not available, no time ( $n$ 24), health constraints ( $n$ 15) and other reasons such as no Internet ( $n 9$ ); however, the majority of the nonparticipants did not state any reason. From the 350 respondents, 281 persons agreed to participate in the pilot study and completed the EFPQ, sixteen accepted initially but failed to complete the EFPQ and an additional four persons were subsequently excluded because of health constraints or non-compliance. Thus, 261 participants returned a completed EFPQ, resulting in a total participation rate of $65.3 \%$. The respective centre-specific participation rates were $62.5 \%$ in EPIC-Florence $(n 50), 68.8 \%$ in EPIC-San Sebastian $(n 55), 70.0 \%$ in EPIC-Potsdam $(n 56)$ and $87.5 \%$ in EGC $(n 70)$. In NOWAC, only $37.5 \%$ ( $n 30)$ of the invited women ( $n$ 80) participated. Overall, participants had a mean age of $55 \cdot 2$ (SD 15.5) years (minimum age: $19 \cdot 4$ years, maximum age: 82.4 years). Demographic and selected lifestyle characteristics of participants and non-participants in the study centres are shown in Table 1. Except in EPIC-Potsdam and EGC, participants were younger than non-participants, but the difference was statistically significant only in EPIC-San Sebastian $(P=0.018)$ and NOWAC $(P=0 \cdot 013)$. In addition, in all centres the proportion of higher-educated subjects was larger in participants than in non-participants (ranged from $10.9 \%$ in EPIC-San Sebastian to $48.3 \%$ in NOWAC), but this difference was not significant. With regard to BMI and smoking status, no consistent pattern across centres was observed: differences in mean BMI between participants and non-participants were only significant in EPIC-San Sebastian $(P=0 \cdot 017)$ and a higher proportion of neversmokers among participants was seen in three of the five centres (except EPIC-Florence and EGC).

Out of the 400 eligible subjects, 252 subjects completed only one recall (63.0\%), 241 completed two $(60.3 \%)$ and 225 three $24-\mathrm{HDR}(56 \cdot 3 \%)$. In all pilot study centres, the completion of the 24-HDR was highest for the first 24-HDR and lowest for the third 24-HDR (Table 2). In EPICFlorence and EGC, the first 24-HDR could be administered among all study participants, whereas in NOWAC only $86.7 \%$ of the women were interviewed. Although completion of the 24-HDR decreased across the recall rounds, the assumption that contact attempts would increase could not be confirmed. The respective mean number of contact attempts was 3.9 (sD $0 \cdot 3$ ), $2 \cdot 8$ (SD $0 \cdot 2$ ) and 2.6 (SD $0 \cdot 4$ ) for the first, second and third 24-HDR, respectively, in EPICSan Sebastian; $1 \cdot 0($ sD $0 \cdot 3), 1 \cdot 1($ sD $0 \cdot 2)$ and $1 \cdot 1$ (sD $0 \cdot 3$ ) in EPIC-Florence; $2 \cdot 4$ (sD 0.3), $2 \cdot 9$ (SD 0.2 ) and 3.6 (sD 0.3) in EPIC-Potsdam; 2.0 (SD 0.3), 1.9 (SD 0.2) and 1.9 (SD 0.3) in EGC and $3.2(\operatorname{sD~} 0 \cdot 5), 2 \cdot 6(\operatorname{sD~} 0.3)$ and $3.2(\operatorname{sD~} 0.5)$ times in NOWAC.

Of the 261 participants enrolled in the pilot study, 143 participants completed the web-EFPQ (54.8\%) and 118 completed the paper-EFPQ $(45 \cdot 2 \%)$. Completion of either the paper-EFPQ or the web-EFPQ differed across centres

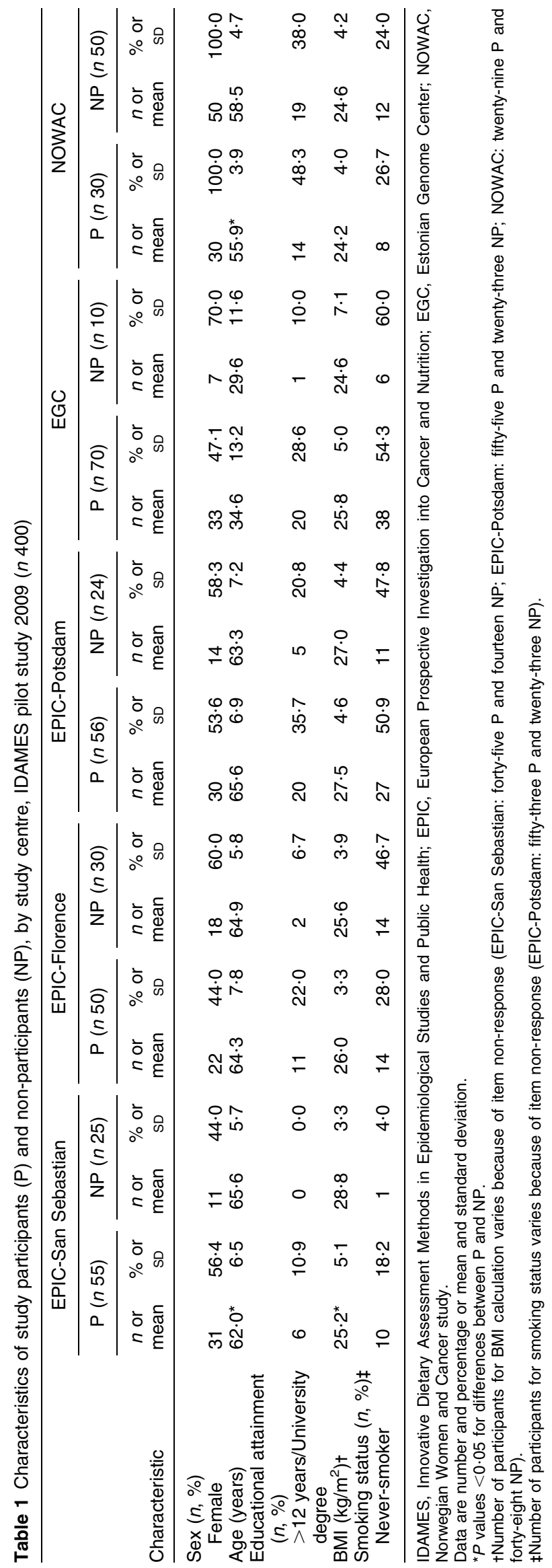


Table 2 Completion of the three 24-HDR among all study participants and by study centre, IDAMES pilot study 2009 ( $n$ 261)

\begin{tabular}{|c|c|c|c|c|c|c|c|c|c|c|c|c|}
\hline \multirow[b]{2}{*}{ 24-HDR } & \multicolumn{2}{|c|}{ All $(n 261)+$} & \multicolumn{2}{|c|}{ EPIC-San Sebastian $(n 55) \ddagger$} & \multicolumn{2}{|c|}{ EPIC-Florence $(n 50)$} & \multicolumn{2}{|c|}{ EPIC-Potsdam ( $n$ 56) } & \multicolumn{2}{|c|}{$\mathrm{EGC}(n 70)$} & \multicolumn{2}{|c|}{ NOWAC $(n 30)$} \\
\hline & $n$ & $\%$ & $n$ & $\%$ & $n$ & $\%$ & $n$ & $\%$ & $n$ & $\%$ & $n$ & $\%$ \\
\hline First & 252 & $96 \cdot 6$ & 51 & $92 \cdot 7$ & 50 & $100 \cdot 0$ & 55 & $98 \cdot 2$ & 70 & $100 \cdot 0$ & 26 & $86 \cdot 7$ \\
\hline Second & 241 & $92 \cdot 3$ & 43 & $78 \cdot 2$ & 49 & $98 \cdot 0$ & 55 & $98 \cdot 2$ & 69 & $98 \cdot 6$ & 25 & $83 \cdot 3$ \\
\hline Third & 225 & $86 \cdot 2$ & 32 & $58 \cdot 2$ & 49 & 98.0 & 53 & $94 \cdot \overline{6}$ & 68 & $97 \cdot 1$ & 23 & $76 \cdot 7$ \\
\hline
\end{tabular}

24-HDR, $24 \mathrm{~h}$ dietary recall; IDAMES, Innovative Dietary Assessment Methods in Epidemiological Studies and Public Health; EPIC, European Prospective Investigation into Cancer and Nutrition; EGC, Estonian Genome Center; NOWAC, Norwegian Women and Cancer study.

tStudy participants were defined as having completed the European Food Propensity Questionnaire.

$\ddagger$ The rounds were not completed because of vacation period.

(presented in Table 3). In EGC, $92.9 \%$ of participants (mean age of $34 \cdot 6$ (SD 13.2) years) chose the web-EFPQ ( $n$ 65). In contrast, there was a preference for paper-EFPQ in EPIC-Potsdam and EPIC-San Sebastian, with 66.1\% ( $n 37)$ and $74.5 \%(n$ 41) completion, respectively. German and Spanish participants were on average 65.6 (SD 6.9) and 61.9 (SD 6.5) years old. In EPIC-Florence, there was no clear preference for one administration mode. Except in EPIC-San Sebastian, web users were significantly younger and had attained a higher education in all centres. Overall, support for completing the web-EFPQ was requested by seven of the 143 participants, whereas ten of 118 persons needed help to answer the paper-EFPQ. The support was provided by telephone, directly in the study centre, or through a visit to the participant's home. In addition, three participants had to complete their web-EFPQ twice because of technical errors. Furthermore, an overall moderate subjective acceptability of the web-EFPQ was found in 109 of 143 webEFPQ users who completed the evaluation questionnaire (EPIC-San Sebastian: $n$ 9, EPIC-Florence: $n$ 20, EPICPotsdam: $n$ 9, EGC: $n 61$ and NOWAC: $n 10)$. In all, $52 \cdot 7 \%$ rated both the clarity of questions and explanations and the visual elements (colours, font, font size, pictures, placing of the elements) and $68 \cdot 8 \%$ considered the user friendliness (clarity, navigation, logical structure) as very good or excellent. In addition, $34 \cdot 1 \%$ indicated having difficulties in reporting their usual food intake, particularly for the intake of fruit, vegetables and alcoholic beverages. The automatically recorded time to complete the webEFPQ (median) ranged from $27 \cdot 0 \mathrm{~min}$ (EGC) and $30 \cdot 8 \mathrm{~min}$ (NOWAC) to $38 \cdot 2 \mathrm{~min}$ (EPIC-Potsdam), $45 \cdot 1 \mathrm{~min}$ (EPICFlorence) and $56.5 \mathrm{~min}$ (EPIC-San Sebastian). Overall, the majority of participants $(52 \cdot 2 \%)$ were logged in for $20-39 \mathrm{~min}$. The percentage of subjects who completed the web-EFPQ within one login session varied from $22 \cdot 2 \%$ (EPIC-San Sebastian) and 36.8\% (EPIC-Potsdam) to $54 \cdot 2 \%$ (EPIC-Florence) and 66.7\% (NOWAC).

\section{Discussion}

The main objective of our pilot study was to investigate the feasibility of applying three 24-HDR interviews conducted by telephone in combination with a uniform, self-administered FPQ in different European countries, making use of Internet technologies. The overall participation rate of about two-thirds suggests that this approach is feasible in the study population and indicates that there is a potential for this approach to be applied in ongoing and new epidemiological studies.

Our findings are in line with one previous American study of combining different assessment instruments in which $60 \%$ of subjects completed four telephoneadministered 24-HDR and one paper-based frequency instrument ${ }^{(8)}$. In the present pilot study, more than half of the eligible subjects completed all three 24-HDR and the EFPQ, whereas almost two-thirds had dietary data from at least two or one 24-HDR and the EFPQ. We found differences in the centre-specific participation rates. Although almost all cohorts share certain common features, such as recruitment of subjects from the general population and the targeted age range of 34-69 years, study designs are distinct. For example, in EPIC-Potsdam, the active 2-year internal follow-up included a second dietary assessment using an FFQ that was mailed to each study participant between 2002 and $2003^{(19)}$. In NOWAC, by contrast, follow-up of exposure information is carried out with approximately 5-year intervals using follow-up questionnaires with four pages asking for detailed information on dietary habits ${ }^{(18)}$, whereas in EGC genes are the primary research topic instead of diet ${ }^{(16)}$. Further details about other pilot study centres can be found elsewhere ${ }^{(20,21)}$. Hence, factors relative to diet that spur subjects to participate may vary across centres, e.g. personal interest in diet or desired weight loss. In our study, personal contact with study staff had also a marked influence on response rates, since the highest participation rate was observed in EGC, in which eligible pilot study participants were recruited during the baseline examination for the local study.

To prevent potential bias introduced by financial incentives or changes in usual dietary habits, our pilot study design implied no financial reward or personalized dietary information for participation, except in EPICFlorence in which a financial reward was offered at the end of data collection that was not mentioned in any other phase of the study. The participation rate may be higher if some well-thought benefits (e.g. personalized feedback, gift card, etc.) are offered to participants ${ }^{(22,23)}$. This issue 
needs consideration, particularly in terms of maintaining long-term compliance in studies with multiple and followup dietary assessments. In addition, 'no time' was the most reported reason for non-participation on the response form; this indicates that improved dietary assessment methods allowing more completion time flexibility could diminish the proportion of non-participants. In general, non-participation was not systematically associated with age, gender, BMI or smoking status; however, in all centres, the proportion of subjects with a higher educational attainment was higher in participants than in non-participants. Overall, the effect of non-participation in approaches of combining different dietary assessment instruments requires further examination in larger study populations.

The advanced statistical methods of combining dietary data from different instruments require repeated 24-HDR, at least two of every individual in the analytical study population, in order to obtain an estimate of intra-individual variance (J Haubrock, U Nöthlings, J-L Volatier et al., unpublished results) ${ }^{(11)}$. A third 24-HDR seems to yield to an increased precision of the individual-level intake estimates, as indicated by unpublished research in a German study population (S Knueppel, personal communication). Similarly, a recent study comparing energy intake from seven 24-HDR with the doubly labelled water method pointed out that, although the first of three recalls was likely to underestimate dietary intake, more than three 24-HDR did not significantly improve the estimation of energy intake $^{(24)}$. Our study adds to the current discussion by showing the feasibility of obtaining dietary data from three short-term measurements from more than $50 \%$ of a culturally diverse European sample. In all study centres, participation was high but decreased slightly with each further telephone interview; in EPIC-San Sebastian, in which the waves could not be completed because of the start of the national summer vacation period before the end of the study, the trend seems to be similar. Given that particularly the third recall appears to be an issue of concern in all centres, the incorporation of additional less-burdensome short-term instruments might be advantageous. In a recent validation study, about $80 \%$ of the 235 enrolled subjects fully completed four measurements of a shorter, self-administered, web-based version of the DASH (Dietary Approaches to Stop Hypertension) questionnaire (DASH Online Questionnaire) that assesses dietary intake over the previous $24 \mathrm{~h}^{(25)}$. By contrast, in another study in undergraduate students, imprecise individual dietary intake estimates were obtained by four measurements with a $1 \mathrm{~d}$ online Food Recall Checklist compared with dietary estimates provided by a $4 \mathrm{~d}$ food diary, although time efficiency of the web-based tool was shown ${ }^{(26)}$. This suggests that a comprehensive 24-HDR computerassisted interview, such as EPIC-SOFT, is still the primary instrument to gather accurate detailed and quantified dietary data, particularly for a standardized assessment. How new self-administered web-based automated 24-HDR 
programmes (e.g. the US Automated Self-Administered 24-Hour Dietary Recall (ASA24; National Cancer Institute, Bethesda, MD, USA) ${ }^{(27)}$ or French MXS (Medical Expert Systems, Paris, France) ${ }^{(28)}$ ) will impact the feasibility of administering repeated recalls in large-scale epidemiological studies remains to be evaluated.

With respect to the feasibility of the web-EFPQ, it needs special mention that our study population was not selected on the basis of Internet access and experience. We approached participants of ongoing cohort studies who were used to classical instruments of data collection such as questionnaires or personal interviews on diet. Therefore, our figures cannot be directly compared with a situation in which study participants are recruited on the basis of Internet access/literacy. According to official European statistics from 2009, Internet access in households differed substantially between European countries, with an average of $65 \%$, reflecting a relatively high access in northern and central countries such as Norway (86\%), Estonia (63\%) and Germany (79\%), but a modest access in the south (e.g. Spain (54\%) and Italy $(47 \%))^{(29)}$. Although not directly comparable to the present study because of the survey methodology, the present completion rates of the web-EFPQ in EPIC-San Sebastian, EGC and NOWAC may therefore partly reflect the country-specific situations regarding Internet penetration, but not for Germany and Italy. Similarly, a population-based study in Sweden - a country in which Internet access is estimated to be about $80 \%$ - reported a lower response rate for a web-based questionnaire with optional questions about dietary habits than for the corresponding paper-and-pencil version ${ }^{(30,31)}$. Thus, further factors should be considered that may influence the response to online questionnaires, beyond the availability of an Internet access. In line with the officially reported highest educational disparities in the regular use of the Internet in the age group of $55-74$ years ${ }^{(29)}$, we found a larger proportion of subjects with a university degree among web-EFPQ users of all centres. In addition, unlike the Swedish study that did not identify differences in response rates by age ${ }^{(30)}$, the present findings showed that in all pilot study centres web-EFPQ users were younger than those who selected to complete the paperEFPQ, in line with results from non-observational studies in middle-aged or elderly study populations ${ }^{(32,33)}$. Age is also likely to contribute to between-centre differences in selecting the web-EFPQ, since study participants from the EPIC cohorts were, on average, older than subjects participating in NOWAC and EGC and may be somehow conditioned to self-report using paper-and-pencil questionnaires about diet. It therefore poses a major challenge to develop and apply easy-to-use and understandable technically advanced tools, tailored to the population under study, in order to reduce selection bias. On the basis of the moderate results of the evaluation questionnaire, we hypothesize further that the current design of the EFPQ might need adaptations in order to be more easily answered by elderly or less-educated subjects. In Native Americans, for example, a simplified design, introductory and HELP screens as well as audio features were advantageous to self-complete a computerized version of the Dietary History Questionnaire ${ }^{(34)}$. Nevertheless, the selfadministered web-EFPQ had the advantage of being able to obtain complete information in a geographically dispersed European study population without questions being skipped, in a reasonable amount of time and with good compatibility with different screen configurations, operating systems and browsers. In addition, the organizational constraints and costs in performing a multi-centre study appeared to be reduced because of the direct and secure electronic data transfer to a central database, automatic control for missing and implausible data and facilitated selfmanagement of the local dietary assessment, consistent with findings from other research ${ }^{(31)}$.

The strength of the present pilot study is that it is the first study investigating the feasibility of combining multiple dietary assessment instruments, including Internet technologies, in a well-characterized multi-centric diverse European study sample, suitable to investigate the feasibility of the approach within a broader perspective. However, major limitations are the limited generalizability and statistical power. All cohorts were not designed to be representative of their countries, although NOWAC is characterized by a high external validity ${ }^{(35)}$. Indeed, EPICSan Sebastian included members of local blood donor associations and employees of selected enterprises; women participating in EPIC-Florence were invited for a local population-based breast cancer screening programme; and EPIC-Potsdam study participants were of higher socioeconomic status and were healthier than the general German population ${ }^{(17,36)}$. Consequently, extrapolation of the present study to general populations should be made with caution. Furthermore, the small sample size may imply that the compliance and ability to complete the EFPQ and the three 24-HDR might be higher in those volunteers who decided to participate in the pilot study than in all the source cohorts, despite the random selection procedure. These aspects could have led to artefactual betweencentre differences regarding the feasibility of the approach. Compared with the original cohorts, participation rates in the present study were substantially higher (e.g. EPICPotsdam originally $22 \cdot 7 \%{ }^{(36)}$, NOWAC originally $\left.57 \cdot 5 \%\right)^{(35)}$. One could infer from the results that this approach might solely be feasible for established cohorts, but the high participation rate in EGC indicates the potential of the approach for newer studies.

In conclusion, the present pilot study design and features of participation showed that the combination of dietary assessment instruments is a feasible approach for assessing diet in different European study populations. For the target age group, it was necessary to offer subjects a paper and an online version of the long-term instrument in order to avoid selection bias. 


\section{Acknowledgements}

The present pilot study was funded by a grant issued by the European Union (no. 2006315 'IDAMES') to explore new avenues for dietary assessment in epidemiological studies and public health (www.idames.eu). None of the authors had a conflict of interest. No supplementary online material has been submitted. A.-K.I., U.H., G.T., S.S., P.A., T.K., D.E., M.B. and H.B. designed the research; A.-K.I., U.H., G.T., S.S., E.B., P.A., T.K., D.E. and M.B. conducted the research; H.W. and N.S. provided essential materials; A.-K.I. and U.H. performed the statistical analysis; A.-K.I., H.W., M.B., G.T., S.S., D.E., T.K., P.A. and H.B. wrote the paper; H.B. had primary responsibility for the final content; D.P., A.M., E.L. and K.W. were members of the IDAMES project involved in the overall research plan. All authors read and approved the final manuscript. The authors thank all the study participants. They also thank everyone who participated actively in the development of the EFPQ and in the field work of the pilot study, as well as those in charge of each centre's technical assistance: Herbert Piechot, Ellen Kohlsdorf, Elektra Polychrondidou (Germany), Paolo Aretini (Italy).

\section{References}

1. Bingham SA, Luben R, Welch A et al. (2003) Are imprecise methods obscuring a relation between fat and breast cancer? Lancet 362, 212-214.

2. Subar AF, Kipnis V, Troiano RP et al. (2003) Using intake biomarkers to evaluate the extent of dietary misreporting in a large sample of adults: the OPEN study. Am J Epidemiol 158, $1-13$.

3. Kristal AR, Peters U \& Potter JD (2005) Is it time to abandon the food frequency questionnaire? Cancer Epidemiol Biomarkers Prev 14, 2826-2828.

4. Thompson FE \& Subar A (2008) Dietary Assessment Methodology. Nutrition in the Prevention and Treatment of Disease, 2nd ed. Oxford: Elsevier, Academic Press.

5. Slimani N, Deharveng G, Charrondiere RU et al. (1999) Structure of the standardized computerized 24-h diet recall interview used as reference method in the 22 centers participating in the EPIC project. European Prospective Investigation into Cancer and Nutrition. Comput Methods Programs Biomed 58, 251-266.

6. Tarasuk V \& Beaton GH (1992) Day-to-day variation in energy and nutrient intake: evidence of individuality in eating behaviour? Appetite 18, 43-54.

7. Beaton GH, Milner J, Corey P et al. (1979) Sources of variance in 24-hour dietary recall data: implications for nutrition study design and interpretation. Am J Clin Nutr 32, 2546-2559.

8. Subar AF, Dodd KW, Guenther PM et al. (2006) The food propensity questionnaire: concept, development, and validation for use as a covariate in a model to estimate usual food intake. J Am Diet Assoc 106, 1556-1563.

9. Illner AK, Nothlings U, Wagner K et al. (2010) The assessment of individual usual food intake in large-scale prospective studies. Ann Nutr Metab 56, 99-105.

10. Kipnis V, Midthune D, Buckman DW et al. (2009) Modeling data with excess zeros and measurement error: application to evaluating relationships between episodically consumed foods and health outcomes. Biometrics 65, 1003-1010.
11. Kipnis V, Shumakovich M \& Midthune D (2009) Combining dietary-assessment instruments in nutritional epidemiology. Presented at 7 th International Conference on Diet and Activity Methods: 2009, Washington, DC, 5-7 June 2009.

12. Beasley JM, Davis A \& Riley WT (2009) Evaluation of a web-based, pictorial diet history questionnaire. Public Health Nutr 12, 651-659.

13. Matthys C, Pynaert I, De KW et al. (2007) Validity and reproducibility of an adolescent web-based food frequency questionnaire. J Am Diet Assoc 107, 605-610.

14. Slimani N, Ferrari P, Ocke M et al. (2000) Standardization of the 24-hour diet recall calibration method used in the European Prospective Investigation into Cancer and Nutrition (EPIC): general concepts and preliminary results. Eur J Clin Nutr 54, 900-917.

15. Noethlings U, Hoffmann K, Bergmann MM et al. (2007) Fitting portion sizes in a self-administered food frequency questionnaire. J Nutr 137, 2781-2786.

16. Metspalu A (2002) Estonian Genome Project - before the take-off and take-off. Bioinformatics 18, Suppl. 2, S152.

17. Riboli E, Hunt KJ, Slimani N et al. (2002) European Prospective Investigation into Cancer and Nutrition (EPIC): study populations and data collection. Public Health Nutr 5, 1113-1124.

18. Lund E, Dumeaux V, Braaten T et al. (2008) Cohort profile: The Norwegian Women and Cancer Study - NOWAC Kvinner og kreft. Int J Epidemiol 37, 36-41.

19. Noethlings U, Hoffmann K, Bergmann MM et al. (2003) Portion size adds limited information on variance in food intake of participants in the EPIC-Potsdam study. $J$ Nutr 133, 510-515.

20. Jakszyn P, Agudo A, Berenguer A et al. (2006) Intake and food sources of nitrites and $N$-nitrosodimethylamine in Spain. Public Health Nutr 9, 785-791.

21. Pala V, Sieri S, Palli D et al. (2003) Diet in the Italian EPIC cohorts: presentation of data and methodological issues. Tumori 89, 594-607.

22. Edwards P, Roberts I, Clarke M et al. (2002) Estimating the effect on incentives in mail survey response rates: a metaanalysis. BMJ 324, 1183.

23. Hunt JR \& White E (1998) Retaining and tracking cohort study members. Epidemiol Rev 20, 57-70.

24. Ma Y, Olendzki BC, Pagoto SL et al. (2009) Number of 24-hour diet recalls needed to estimate energy intake. Ann Epidemiol 19, 553-559.

25. Apovian CM, Murphy MC, Cullum-Dugan D et al. (2010) Validation of a web-based dietary questionnaire designed for the DASH (Dietary Approaches to Stop Hypertension) diet: the DASH online questionnaire. Public Health Nutr 13, 615-622.

26. Comrie F, Masson LF \& McNeill G (2009) A novel online Food Recall Checklist for use in an undergraduate student population: a comparison with diet diaries. Nutr J $\mathbf{8}, 13$.

27. Subar AF, Thompson FE, Potischman N et al. (2007) Formative research of a quick list for an automated selfadministered 24-hour dietary recall. J Am Diet Assoc 107, 1002-1007.

28. Hercberg S, Castetbon K, Czernichow S et al. (2010) The Nutrinet-Santé Study: a web-based prospective study on the relationship between nutrition and health and determinants of dietary patterns and nutritional status. BMC Public Health 10, 242.

29. Lööf A \& Seybert H (2009) Internet usage in 2009 households and individuals. http://epp eurostat ec europa eu/cache/ITY OFFPUB/KS-QA-09-046/EN/KS-QA-009-046EN pdf 2010 (accessed December 2009).

30. Balter KA, Balter O, Fondell E et al. (2005) Web-based and mailed questionnaires: a comparison of response rates and compliance. Epidemiology 16, 577-579. 
31. Ekman A, Dickman PW, Klint A et al. (2006) Feasibility of using web-based questionnaires in large population-based epidemiological studies. Eur J Epidemiol 21, 103-111.

32. Klovning A, Sandvik H \& Hunskaar S (2009) Web-based survey attracted age-biased sample with more severe illness than paper-based survey. J Clin Epidemiol 62, 1068-1074.

33. Verheijden MW, Jans MP, Hildebrandt VH et al. (2007) Rates and determinants of repeated participation in a web-based behavior change program for healthy body weight and healthy lifestyle. J Med Internet Res $\mathbf{9}$, e1.
34. Edwards SL, Slattery ML, Murtaugh MA et al. (2007) Development and use of touch-screen audio computer-assisted self-interviewing in a study of American Indians. $\mathrm{Am} \mathrm{J}$ Epidemiol 165, 1336-1342.

35. Lund E, Kumle M, Braaten T et al. (2003) External validity in a population-based national prospective study - the Norwegian Women and Cancer Study (NOWAC). Cancer Causes Control 14, 1001-1008.

36. Boeing H, Korfmann A \& Bergmann MM (1999) Recruitment procedures of EPIC-Germany. European Investigation into Cancer and Nutrition. Ann Nutr Metab 43, 205-215. 